\title{
The Fourth Revolution: Philosophical Foundations and Technological Implications
}

\author{
Hilmi Demir
}

Received: 15 June 2010 / Accepted: 29 July 2010 / Published online: 28 July 2010

(C) Springer Science+Business Media B.V. 2010

\begin{abstract}
This article introduces this special issue of Knowledge, Technology and Policy. It also explains why Luciano Floridi's Philosophy of Technology is chosen as the topic of the special issue.
\end{abstract}

Keywords Information Age · Information revolution · Philosophy of Information

The age we live in is dominated by information and communication technologies. Computers, cell phones, iPods, and social internet networks are just some of these technologies without which life as we know would be quite different. Because of these technologies, the age we live in is usually called the "Information Age." Although the exact meaning of this phrase significantly varies from one usage to another, we all more or less understand it. It refers to an age when computers have become an essential part of our daily lives. Therefore, it would not be wrong to claim that the information age started with the invention of computers. This takes us to the 1940 s as more or less the starting point of the information age. The changes that have happened since then have brought about new issues, questions, challenges, and opportunities that need to be carefully and scholarly analyzed for the betterment of humanity. One category of these new issues is knowledge-related. Our knowledge has expanded significantly since the information revolution. Whether this expansion is just a quantitative change or if it points to a paradigmatic shift in our understanding of the universe and ourselves is an important question that needs to be answered. The other category is policy-related questions and challenges. With the introduction of the Internet, there are new ways of community building through social networks. Knowledge acquisition and dissemination are now accessible to all people in the world, at least potentially. This brings about new challenges, particularly regarding the freedom of expression. Likewise, with the information and communication technologies, new and more efficient ways of bringing governmental and administrative services to the general public have become

\footnotetext{
H. Demir $(\bowtie)$

Bilkent University, Ankara, Turkey

e-mail: hilmi@bilkent.edu.tr
} 
possible. How we implement such technologies for these services is not just a matter of technicality; the implementation could have far reaching consequences, sometimes positive (e.g., a more transparent public administration) and sometimes negative (e.g., constant surveillance of citizens). Thus, not only bureaucrats but also scholars need to be involved in designing new policies. The last category is technology-related. Information and communication advancements have widened the potential of new technologies beyond imagination. Almost any technology that could be imagined seems to be empirically possible now, from artificial minds to telepathy through brain waves. Soon enough, artificial agents will start interacting with us, and such interaction may not be qualitatively different than our interaction with other human beings. Soon enough, the offline/online distinction, which is already blurring, will disappear. Surely enough, such changes raise new ethical questions. Should we include these man-made agents into the domain of moral evaluation? If yes, how are we to evaluate artificial agents morally?

In this short paragraph, I have listed just a few of the new issues, questions, and challenges brought about by the information revolution. As mentioned, these need to be carefully analyzed if we want a better future for humanity. After all, nobody wants to experience a new Hiroshima, a despicable act that was made possible with new technological inventions. Thus, scholarly scrutiny is of paramount significance. This special issue aims to do just that. It does so, however, in a specific context. The context is Luciano Floridi's Philosophy of Technology, which is a part of his general framework of the Philosophy of Information. Before I proceed with the articles in this special issue, I need to specify why Floridi's framework has been chosen as the context in which some of the above mentioned issues are analyzed and discussed.

Luciano Floridi, since his talk, "Should there be a Philosophy of Information," given at King's College (Floridi 2010b), has been constructing a new framework, a new way of doing philosophy that could address the needs and issues that the information revolution brought about. In his new framework, he calls the information revolution the fourth revolution, following the Copernican, Darwinian, and Freudian revolutions. $\mathrm{He}$ analyzes these four revolutions with respect to their introvert effects, i.e., their effects on our understanding of ourselves (Floridi 2010a, p. 8). Before Copernicus, humans, at least in the Western world, thought that our planet was special among all other astronomical bodies; it was thought to be the center of the universe. The Copernican revolution changed that; the effect was the loss of the privileged position of our planet in the universe. The disappointment caused by this loss, however, was compensated for by another privilege. Human beings were special among all other creatures that inhabit the world. This comforting thought continued until Darwin, and another disappointment was awaiting us. With Darwin, we lost our privileged position among other living creatures. After all, evolution is a continuous process from animals to humans. However disappointing Darwin's news was, we found solace in another privilege. Only we human beings have the control of our mentality; other animals do not. Alas, not long after Darwin, Freud made us realize that we did not even have that privilege of knowing and controlling our mental world. The pattern continues: once again a disappointment and once again another comforting privileged position. For now, mankind is in a more special position, at least, than inanimate beings, natural or artificial. Luciano Floridi claims that because of the fourth revolution, this may not 
always be so. Once again, according to Floridi, it is time to reexamine our understanding of the universe and ourselves.

What we are currently experiencing is therefore a fourth revolution, in the process of dislocation and reassessment of our fundamental nature and role in the universe. We are modifying our everyday perspective on the ultimate nature of reality, that is, our metaphysics, from a materialist one, in which physical objects and processes play a key role, to an informational one...the criterion of existence-what it means for something to exist-is no longer being actually immutable...or being potentially subject to perception...but being potentially subject to interaction, even if intangible. To be is to be intractable, even if the interaction is only indirect (Floridi 2010a, p. 12).

With the fourth revolution, we realize that human nature is intrinsically informational. And with respect to being informational entities, we are not much different than other entities, be it natural or artificial. As Floridi claims, this is a very radical shift in our understanding of universe and ourselves. But, at least in my opinion, the gist of his claim is correct. After all, the information revolution itself is radical, and it is only normal that such a radical revolution would lead to a very radical shift in our understanding of the universe.

On the basis of his analysis of the fourth revolution, Floridi has constructed a general framework for the Philosophy of Information. In his framework, he defines the content and the boundaries of the Philosophy of Information as a subfield of philosophy. He has also identified the open problems of the field for future research. In that respect, he single handedly defined the field. Although the Philosophy of Information as a field of inquiry could historically be traced back to Leibniz, it would not be wrong to claim that the field acquired its legitimate status among the other subfields of philosophy with Floridi's work.

Floridi's framework not only defines the content and the boundaries of Philosophy of Information as a field of inquiry but also provides novel approaches and ideas for a wide range of philosophical issues, ranging from Ethics to Logic (his Information Ethics and Informational Logic), from Ontology to Epistemology (his Informational Epistemology and Informational Metaphysics), from Semantics to Philosophy of Mind.

As mentioned above, the overall goal of this special issue is to provide a venue for analyzing philosophical foundations and technological implications of the information revolution. This is done in the context of Floridi's Philosophy of Information. I believe that I have stated two good reasons for choosing Floridi's framework as the context of this issue. First, his framework is the first attempt to establish Philosophy of Information as a legitimate and independent field of inquiry. Second, his framework offers novel approaches, which are based on the notion of information, for a wide range of philosophical issues.

Now let me briefly state the content of each contribution to this double special issue. In total, we have 13 articles. The first issue starts with Donald Gillies's contribution. In his article, Gillies examines Floridi's informational structural realism within the context of Philosophy of Mathematics. He claims that mathematical realism is a special case of informational realism. He agrees with Floridi about informational realism, but he proposes a constructive Aristotelian 
version of informational realism as opposed to Floridi's version, which is more Platonic.

The issue continues with Patrick Allo's article, "A Classical Prejudice." Allo analyzes the relation between dialetheism, i.e., the view that there are true contradictions, and the veridicality thesis, the fundamental thesis of Floridi's semantic theory of information. The veridicality thesis states that semantic information has to be true. In his analysis, Allo claims that this thesis is compatible with dialetheism. In doing that, he, to my knowledge for the first time in the literature, points out a distinction between the veridicality thesis and the non-falsity thesis, i.e., that information cannot be false.

The third article is a criticism of Floridi's Philosophy of Information by Pieter Adriaans. Adriaans claims that the formal treatment of the notion of information as a general theory of entropy, as developed in physics, information theory, and computer science, is sufficient for explaining philosophical notions such as knowledge, meaning, and truth. If this is the case, however, Floridi's semantic theory of information becomes superfluous. This is exactly what Adriaans claims: Floridi's Philosophy of Information is unnecessary. Although one may disagree with Adriaans, as Floridi does (see his reply piece at the end of the second volume), his article is an important contribution, particularly because it emphasizes the possible philosophical value of the formal treatment of the notion of information. I believe that since the beginning of semantic treatments of information (Bar-Hillel and Carnap 1953; Bar-Hillel 1964), the philosophical importance of the formal treatment of information based on the notion of entropy has been undervalued. Thus, Adriaans' article tries to direct our attention to this undervalued notion.

Jean-Gabriel Ganascia, in his "Epistemology of AI Revisited in the Light of the Philosophy of Information," analyzes the epistemological foundations of Artificial Intelligence on the basis of the distinction between "sciences of nature" and "sciences of culture" and claims that AI cannot be reduced to either of these because AI is an "intermediary domain." He further claims that Floridi's notion of "levels of abstractions" is useful for understanding basic dynamics of this intermediary domain.

The fifth article in the first issue is Tomasso Piazza's "Perceptual Evidence and Information." In Floridi's Philosophy of Information, knowledge is analyzed as accounted information. According to Piazza, in Floridi's understanding of knowledge as accounted information, there is no room for justification and/or evidence. $\mathrm{He}$ agrees with Floridi's general motivation, but finds this conclusion too drastic. He argues for a less drastic conclusion, in which information is used to explain perceptual evidence and then perceptual evidence is used to explain the notion of knowledge.

Flavio Soares Correa da Silva's "On the Ethics of Democratic Access to Web Information: Some Reflections on Recent Work by Luciano Floridi" is an excellent analysis and combination of Floridi's Information Ethics, Information Logic, and his work on technological developments. The ultimate aim of Correa da Silva's analysis is to provide sound philosophical foundations for establishing more democratic programs for Electronic Governments. In that respect, his contribution shows the importance of philosophical analysis for more democratic policies.

The last article in the first issue is Joseph Brenner's “A Logic of Ethical Information." In his article, Brenner points out similarities and differences 
between his "Logic in Reality," which is an extension of logic to complex real processes, and Floridi's Philosophy of Information. He claims that "Logic in Reality" provides solutions for some of the open problems of Philosophy of Information.

The first article in the second issue is Michael Byron's "Floridi's Fourth Revolution and the Demise of Ethics." Michael Byron starts his article with an observation about the difference between the fourth revolution and the previous three revolutions (Copernican, Darwinian, and Freudian). He claims that the first three were a result of a finding that led to a reconceptualization of human nature, whereas the fourth revolution is not a result of a finding about the universe or ourselves; rather, it is a result of a making (i.e., computers made by humans). He, similar to Floridi, finds mainstream ethical theories insufficient for explaining the ethical issues of the fourth revolution, mainly because of the difference just mentioned. In contrast to Floridi, however, he claims that Floridi's Information Ethics is also unsatisfactory for the ethical issues brought about by the fourth revolution.

The second issue continues with Massimo Durante's "The Value of Information as Ontological Pluralism." In this article, Durante turns our attention to an interesting feature of Floridi's Information Ethics, which is its consistency with a pluralistic notion of Being.

The third article is Tony Doyle's contribution, "A Critique of Information Ethics," in which he criticizes Floridi's Information Ethics. One of the important features of Floridi's Information Ethics is that it expands the domain of moral evaluation to the extent that the domain includes all possible informational entities. Doyle presents arguments against the expansion of the moral domain. He also presents examples in which Consequentialism fares better than Floridi's Information Ethics.

Wolfgang Hofkirchner's “How to Design Infosphere: The Fourth Revolution, the Management of the Life Cycle of Information, and Information Ethics as a Macroethics" is a constructive criticism of Floridi's notion of the infosphere and his Information Ethics. Hofkirchner tries to summarize the main gist of Floridi's Philosophy of Information in three main claims. Then, he analyzes and suggests revisions for each. His suggested revisions are based on several different notions, some of which are noogenesis, collective intelligence, and friction in social and physical aspects.

The fifth article in the second issue is Orlin Vakarelov's "Pre-Cognitive Semantic Information." Vakarelov analyzes one of the fundamental problems of the Philosophy of Information, i.e., how semantic information emerges from the underlying physical processes, from a dynamical systems perspective. He suggests his own solution for the problem as an expansion of Floridi's Semantic Information.

The next contribution is a nice interdisciplinary article by Gunji, Niizato, Murakami, and Tani. They use findings from zoology to solve some of the open problems of the Philosophy of Information, especially for the symbol grounding problem. The key in their solution is their interesting concept of Type-Ken, which is a combination of Type and Token concepts.

Lastly, the second issue ends with Luciano Floridi's replies to the articles published in this double special issue. 


\section{References}

Bar-Hillel, Y. (1964). Language and information: Selected essays on their theory and application. Reading: Addison-Wesley.

Bar-Hillel, Y., \& Carnap, R. (1953). An outline of a theory of semantic information. Rep. in Bar-Hillel [1964], pp. 221-274.

Floridi, L. (2010a). Information: A very short introduction. Oxford: Oxford University Press.

Floridi, L. (2010b). The philosophy of information. Oxford: Oxford University Press. 